\title{
Comparisons of reaction time patterns in a sequential visual recognition task with simple geometric forms
}

\author{
WALLACE T. CLEAVES \\ Institute of Child Development, University of Minnesota, Minneapolis, Minnesota 55455
}

\begin{abstract}
Right isosceles triangles, which differed in size and orientation, were presented in a sequential visual recognition task. Subjects produced an identity match response if the first self-presented stimulus, now in memory, was identical to a sequentially presented stimulus and a nonidentity match response if the subsequently presented stimulus differed in either or both size and orientation. The pattern of nonidentity recognition reaction times produced was compared to predicted patterns deduced from several theories of form information processing. The pattern of responses for both group and individual data were better described by serial and parallel self-terminating models with probabilistic assumptions than they were by template or deterministic serial or parallel models. A second study, in which identity and nonidentity response bias was equated, found that the identity responses were significantly faster than nonidentity responses, supporting wholistic processing for the identity matches and, when combined with the nonidentity match results, supporting a dual process theory.
\end{abstract}

Studies of recognition memory have frequently used the same-different comparison task in which two or more stimuli are compared on predefined criteria. Usually the subject's decision reaction time and errors are the primary dependent measures. In recent years there have been significant advances in identifying the processes underlying recognition memory using this technique. Most researchers seem to show a growing conviction that the different response or nonidentity match is based on a feature comparison process (e.g., Egeth, 1966; Hawkins, 1969; Nickerson, 1967; Silverman \& Goldberg, 1975).

The conclusion that the mental comparison which results in a different response is based on feature analysis may be premature. We believe many of these studies might have created experimental demands that forced feature abstraction strategies and therefore did not provide an adequate test of potential wholistic image or template processing. Some studies have used pairs of simultaneously presented stimuli (e.g., Hock, 1973; Shepard \& Metzler, 1971) where the subject might have cross-checked specific features until a match or mismatch was determined.

The study reported here is based on a dissertation submitted to the graduate school, University of California, Riverside, in partial fulfillment of the requirements for the PhD degree. This research was supported, in part, by a Chancellors Patent Fund Grant to the author from the University of California. The author gratefully acknowledges the guidance provided by David H. Warren, his dissertation advisor, and wishes to thank Edgar C. Howell for his assistance in the analysis of the data.
Others have used stimuli either composed of many subelements (e.g., Silverman, 1973) or of such complexity (e.g., Hock, 1973; Shepard \& Metzler, 1971) that it seems improbable that the subject could have held a veridical wholistic image in memory. Even when studies have used relatively simple stimuli in a sequential recognition task, the stimuli have usually been subassemblies of separable stimulus units (e.g., Downing \& Gossman, 1970; Egeth, 1966), such as a square with a separate bar placed across it, where there might have been an experimental demand to treat each subunit as a separate attribute or feature.

The possibility that visual form stimuli can be utilized as wholistic images is supported by Posner's (Posner, Boies, Eichelman, \& Taylor, 1969) evidence for the visual character of letter form images, Shepard and Chipman's (1970) finding of a "secondorder" isomorphism for the internal representation of images of the shapes of U.S. states, and Shepard and his colleagues' description of analog processes even more complex than Posner's on generated visual images (Cooper, 1975; Cooper \& Shepard, 1973; Kosslyn, 1975). With such strong evidence for the existence of useful visual imagery, it would seem logical that the recognition response in memory tasks could be based on a template comparison.

\section{EXPERIMENT 1}

To determine if template processing was possible in a same-different decision task, we attempted to maximize conditions which were thought to be favor- 
able to wholistic or template processing and then to perform an analysis of the results against a wider range of template models than had previously been tested. In order to force subjects to retain information in memory about the whole figure, the recognition task was done sequentially. To maximize the ability of subjects to retain an image, a small constrained set of simple stimuli was utilized. In order to assure that the stimuli would be treated as wholes, the selection of dimensions upon which the stimuli could be discriminated, size and orientation, were integral and inseparable attributes of the forms in Garner's sense (Garner \& Felfoldy, 1970), neither dimension being removable without destroying the stimulus. The stimuli to be compared were projected sequentially exactly onto the same surface, decreasing the need for eye movements or scanning.

\section{Method}

In overview, subjects presented the figure to be memorized (memory figure) to themselves and then, after a 0.5 -sec interstimulus interval, the figure to be compared (test figure) to the figure in memory was presented to them. As soon after presentation of the test figure as possible, the subject was to verbally respond "yes" if the figure held in memory and the test figure were identical and "no" if the stimuli differed in either size or orientation, or both.

\section{Stimulus Selection}

All stimuli were right isosceles triangles which differed only in size and orientation. The seven stimuli utilized are shown in Figure 1. Using each of these seven stimuli as memory and test forms, each stimulus against every other stimulus, creates a 7 by 7 , or 49 , cell comparison matrix with 7 cells (on the diagonal) being "yes," or identity, and the other 42 being "no," or nonidentity, pairings. Of the $\mathbf{4 2}$ nonidentity pairings, 22 differ only in orientation, 16 in size and orientation, and 4 in size only. Therefore, 38 of the 42 pairings differ in orientation, while 20 of the 42 differ in size. The larger forms had exactly twice the surface area of the smaller forms, and their sides were equal in length to the hypotenuse of the smaller forms.

\section{Subjects}

The subjects were volunteers from undergraduate courses at the University of California, Riverside. They received extra credit in courses for their participation in the experiment. Only subjects who had normal or corrected vision in both eyes and had no known neurological or sensory impairments were utilized. The subjects ranged in age from 17.0 to 26.9 years; the mean age was 19.8 years.

Although 26 students were tested, only the data of 24 were analyzed. The data of two students were withdrawn because, in statements in their postsession questionnaires, they indicated that they had not performed the tasks as requested.

\footnotetext{
Apparatus

The subject sat on a standard chair before a panel which extended from waist height to above horizontal eye level. A circular 12.5-cm-diam rear-projection ground-glass screen was located on the subject's midline, $45 \mathrm{~cm}$ from the bridge of his nose. The screen was inclined and centered, to be perpendicular to the subject's resting line of sight, $15^{\circ}$ below horizontal eye level. Directly below the projection screen was a circular opening containing a metal plate connected to a proximity contact sensor switch. Placed below the contact plate opening was a small shelf designed to provide the subject with a handrest between trials.
}
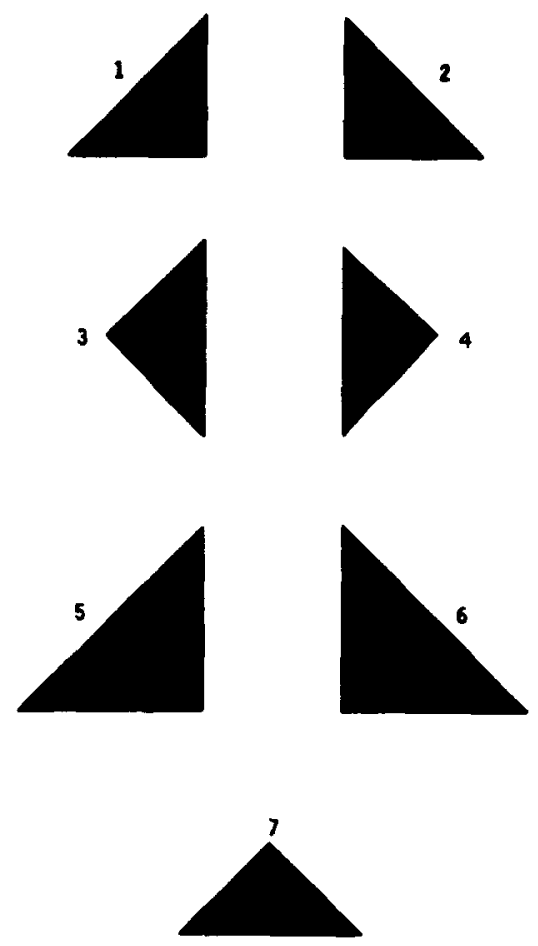

Figure 1. The stimuli.

The stimuli were all projected onto the ground glass screen and subtended between 5.08 and $10.08 \mathrm{deg}$ of visual angle. Separate projectors were used for presentation of the memory and test stimuli. Both projectors utilized glass-mounted slides and projected to the same location through an optical cube to minimize angular distortion and equalize spectral color absorption. The transilluminated intensity of the projections at the screen's surface was $50.6 \mathrm{~lx}$, and the general room illumination was $64.1 \mathrm{~cd} / \mathrm{m}^{2}$. The image shift variance between the memory and test projections of the forms was $2.1 \mathrm{~mm}$.

\section{Procedure}

The subject placed one hand in his lap and rested his dominant hand on the shelf. After a ready signal from the experimenter, he moved his hand off the shelf to the contact plate; this projected the memory figure for study. He was requested to present the stimulus, by keeping his hand in contact with the plate, just long enough to do the subsequent recognition task without error. Pilot work indicated that a subject's reaction time was significantly shorted and less variable if a proximity contact sensor plate, rather than a mechanical switch or key, was used.

When the subject removed his hand from the contact plate, projection of the memory figure ceased, and after a $500-\mathrm{msec}$ blank screen interval, the test figure was automatically projected. Under these presentation conditions it was expected that the afterimage would fade out before the test figure was presented.

The subject was instructed to say the word "yes" into a microphone, connected to a voice switch and a millisecond timer, if the projected test figure was the same size and orientation as the memory figure and "no" if the figures differed in either size, or orientation, or both. Subjects were requested to tell the experimenter if they felt their recognition response had been in error.

Prior to beginning the data collection, the experimenter demonstrated the testing sequence. Each subject went through 14 randomly selected practice trials, designed to give the subject experience with the basic types of size and orientation differences, as well as practice trials with identity responses. After 
completion of the familiarization period, the subject was told that actual data collection would begin. In fact, a series of criterion trials was started; these trials were taken until the subject had made seven consecutive correct responses, at least one of which was a "yes," or identity, match. Only 4 of the 24 subjects made an error during the criterion run and only 1 made two errors.

After the criterion was met, the actual data trials were begun without pause. Two complete randomly ordered blocks of the 49 possible memory-test pairings of the seven stimuli were presented; subjects completed one block prior to beginning the next. Any trial in which an error was made was retaken at the end of that block of trials. There were no pauses between the regular and repeated trials, and subjects were not informed that any trials were being retaken. On an average, the subjects made only $1.06 \%$ errors over the 98 trials. With instructions, practice, and actual data collection, a session took an average of $66 \mathrm{~min}$ to complete.

\section{Results and Discussion}

On each trial, two times were recorded: the time the subject presented the memory figure for study and the time it took to make the verbal response to the test figure. Any trial in which a subject made an error in recognition was replaced by the appropriate retaken trial as outlined above.

\section{Memory Figure Study Time}

The amount of time the subjects presented the memory figures to themselves was entered into an analysis of variance with two factors: memory figure and trial block. Only the effect of trial block was significant, $F(1,2315)=500, p<.01$. On the first block of trials, the subjects projected the memory figures for an average of $355 \mathrm{msec}$; this dropped to $291 \mathrm{msec}$ on the second block, a decrease of $19.8 \%$. The significant decrease in study time between blocks may indicate a refinement or a change in the subject's processing strategy. These possibilities will be further investigated by a comparison between the reaction time patterns produced in the first and second trial blocks.

\section{Test Figure Decision Time}

Table 1 presents the reaction times in milliseconds averaged across the subjects for each replication or block of the 49 memory-test form pairings. Each row of the matrix presents the average decision reaction time to each test form for a specific memory form.

Decision response times. Verbal reaction times of the "yes" and "no" responses to a neutral grid pattern stimulus were taken for each subject both before and after the session. These times were averaged and subtracted out of the subjects' decision response times to remove the difference in voice-key triggering speed between the verbal responses. In the analysis of the resulting data, it was found that the average corrected "yes" response of $204 \mathrm{msec}$ was significantly slower than the average "no" response speed of $177 \mathrm{msec}, \mathrm{F}(1,46)=8.81, \mathrm{p}<.01$. This finding is the oppposite of the results of most other studies (e.g., Bamber, 1969). Behar's finding
(1963) that response probabilities can bias the subject toward faster responding to the more frequently required response indicates that the six to one ratio of "no" to "yes" responses in this study quite possibly created a bias in favor of faster nonidentity responding. This concern motivated the running of Experiment 2, reported later in this paper, where the number of identity and nonidentity responses is equated.

Nonidentity response times. Examination of the balanced set of forms $1,2,5$, and 6 provides for analysis between stimulus pairs which differ only in size (1-5, 5-1, 2-6, and 6-2), only in orientation (1-2, $2-1,5-6$, and $6-5)$, or in both size and orientation (1-6, 6-1, 2-5, and 5-2). The decision response times of these pairs for all subjects were entered into an analysis where the dimension of difference, specific memory and test form, and trial block were all within-subject factors. Only the dimension of difference, $F(2,529)=12.95, p<.01$, and trial block factors, $F(1,529)=35.85, p<.01$, were statistically significant.

Scheffé post hoc comparison on the across-trial block summary found that forms which differed from each other in both size and orientation were responded to significantly faster than those that differed only in size, $F(2,529)=12.07, p<.01$, or only in orientation, $F(2,529)=6.52, \mathrm{p}<.01$. The analysis between the size-only and orientation-only pairings was not significant, $F(2,529)=0.85$, $\mathrm{p}>.42$. It took subjects an average of only $533 \mathrm{msec}$ to respond if the form held in memory and the test stimulus differed in both size and orientation, but it took an average of $571 \mathrm{msec}$ if they differed only in size and $561 \mathrm{msec}$ if they differed only in orientation. Since the size- and orientation-only decision speeds are comparable, it is apparent that they are

Table 1

Raw Data Matrices

\begin{tabular}{|c|c|c|c|c|c|c|c|}
\hline \multirow{2}{*}{$\begin{array}{c}\text { Memory } \\
\text { Form }\end{array}$} & \multicolumn{7}{|c|}{ Test Form } \\
\hline & 1 & 2 & 3 & 4 & 5 & 6 & 7 \\
\hline & \multicolumn{7}{|c|}{ Trial Block 1} \\
\hline 1 & 574 & 560 & 571 & 569 & 596 & 557 & 566 \\
\hline 2 & 591 & 610 & 550 & 551 & 533 & 578 & 587 \\
\hline 3 & 577 & 559 & 626 & 578 & 551 & 575 & 551 \\
\hline 4 & 572 & 571 & 556 & 572 & 577 & 555 & 569 \\
\hline 5 & 580 & 579 & 565 & 537 & 557 & 584 & 537 \\
\hline 6 & 545 & 588 & 576 & 568 & 586 & 590 & 570 \\
\hline \multirow[t]{2}{*}{7} & 557 & 556 & 563 & 571 & 555 & 539 & 595 \\
\hline & \multicolumn{7}{|c|}{ Trial Block 2} \\
\hline 1 & 539 & 536 & 526 & 514 & 578 & 538 & 510 \\
\hline 2 & 532 & 559 & 526 & 518 & 489 & 560 & 522 \\
\hline 3 & 541 & 535 & 595 & 541 & 526 & 508 & 515 \\
\hline 4 & 515 & 522 & 528 & 517 & 496 & 508 & 536 \\
\hline 5 & 526 & 503 & 531 & 526 & 520 & 562 & 507 \\
\hline 6 & 524 & 555 & 517 & 518 & 530 & 537 & 497 \\
\hline 7 & 537 & 530 & 537 & 517 & 504 & 500 & 540 \\
\hline
\end{tabular}


not processed in a fixed order. Why one feature would be selected before the other for processing on only some trials is unclear.

This analysis clearly shows that pairs that differed in two ways are discriminated faster than pairs differing only in one. In fact, the fastest response time in six of the seven rows is for pairs differing in both size and orientation. Unfortunately this analysis is designed only as a test of feature processing and tells us nothing about the potential descriptive ability of template models.

\section{MODEL TESTING}

Part of the purpose of this report was to develop a model testing procedure which would overcome some of the concerns we had about how reaction time data are handled in most studies. We wished to develop a procedure which not only provided a method to determine the model which best described the subject's data, but also would provide for model building based on the results of the analysis. We decided to work with clusters of predictions in a manner similar to Townsend's (1971a) analysis of visual form confusion data and Geyer and DeWald's (1973) stress analysis model testing procedure.

The technique we developed is based on a simple Pearson product moment correlation where we ask how closely the pattern of decision reaction times of subjects matches the predicted pattern of reaction times for each theory being tested. Correlation has seldom been used for model testing in the past because the form of the model predictions which are often simple ordinal ranks between cells with lots of ties or nonpredictions and the raw millisecond reaction times which are assumed to be based on an underlying interval scale are not linearly related, making such an approach nonsensical. The technique we have outlined below attempts to overcome this deficit by transforming the raw reaction times of subjects into the same ordinal form as the models without losing the interval value of the subject's decision reaction times. This is accomplished by converting the subject's decision reaction time matrix into a family of ordinally ranked matrices where the ranking decision rule is well defined and the rankings are based on the interval differences present in the reaction time matrix.

\section{Theory to Model Development}

The current trend toward multistate decision models makes separate analysis of identity and nonidentity responses methodologically palatable, if not mandatory. We wished to separately examine the 42 nonidentity response cells of the 7 by 7,49 -cell, martrix of subjects as a whole, comparing the ob- tained pattern of "no" reaction time responses against hypothetical patterns predicted by various theories. For our starting point, we decided to test the basic models described by Neisser (1967). We did not expect any specific model tested to be an exact match to the observed data for this rather specific experimental context, but by a series of successive approximations, starting at this simplified level, we hoped to narrow the field to a more manageable set of descriptive models.

Before any comprehensive investigation of theories could be attempted, it was necessary to define them in operational terms. Table 2 presents the list derived and the columns contain the branch points which conceptually or operationally differentiate one theory from another when applied to our stimuli. The main purpose of this listing was to define the selected theories sufficiently to make predictions based on the stimuli used in this study. It was soon realized that most of the theories were too generally stated. It became necessary to define several models for each theory to adequately represent them. When completed, the list had grown to 21 models, 15 of which create unique models or matrices of predictions using our stimuli.

In the index number column of Table 2 are listed model numbers. These numbers are used to simplify discussion of the models in the text of the report. Different theories which derived the same matrix of predictions are identified by a letter in the far right column; those with the same letter produced the same set of predictions. Following is a description of the theories presented in Table 2. (A set of tables giving the exact quantitative differences between cells by theory can be obtained from the author.)

Template-mute. In this model, the stimuli are processed as functional wholes. In the recognition comparison decision, differences between the figure in memory and the test figure influence the error rate of subjects but not the speed of the response. This model is essentially nonpredictive, or mute, and states that no consistent pattern of reaction times should be observable. Both this model and all others with a group index of $\mathrm{D}$ are nonpredictive, and as such are not adequately tested by our correlational approach, since they contain no differential rank predictions and therefore cannot show corelatedness. They are tested in that other models which show strong descriptive ability for the subject's reaction time patterns allow us to reject the null hypothesis which these models represent.

Template-analog. In Neisser's (1967) formulation, template models are wholistic in character and in this strict interpretation are mathematically the limiting case of feature testing. It is assumed that reaction time is related to some higher order single complex feature upon which the stimuli are 
Table 2

Theory and Model List

\begin{tabular}{|c|c|c|c|c|c|c|}
\hline $\begin{array}{c}\text { Model } \\
\text { Type }\end{array}$ & Subtype & Process & Subprocess & Subprocess & $\begin{array}{c}\text { Model } \\
\text { Number }\end{array}$ & $\begin{array}{l}\text { Group } \\
\text { Index }\end{array}$ \\
\hline & Mute & & & & 11000 & D \\
\hline Template & Analog & Centered & $\begin{array}{l}\text { Largest area } \\
\text { Largest area } \\
\text { Total area } \\
\text { Total area } \\
\text { Mean area } \\
\text { Mean area }\end{array}$ & $\begin{array}{l}\text { Literal } \\
\text { Modified } \\
\text { Literal } \\
\text { Modified } \\
\text { Literal } \\
\text { Modified }\end{array}$ & $\begin{array}{l}12110 \\
12111 \\
12120 \\
12121 \\
12130 \\
12131\end{array}$ & \\
\hline \multirow{4}{*}{ Feature } & Parailel & & $\begin{array}{l}\text { Exhaustive } \\
\text { Self-term } \\
\text { Self-term } \\
\text { Self-term }\end{array}$ & $\begin{array}{l}0<S / S<0 \\
S<0 \\
0<S \\
S=0\end{array}$ & $\begin{array}{l}21010 \\
21021 \\
21022 \\
21023\end{array}$ & $\begin{array}{l}\mathbf{D} \\
\mathbf{B} \\
\mathbf{A} \\
\mathbf{D}\end{array}$ \\
\hline & \multirow{3}{*}{ Serial } & Memory & $\begin{array}{l}\text { Self-term A } \\
\text { Self-term A } \\
\text { Self-term B }\end{array}$ & $\begin{array}{l}S<0 \\
0<S \\
0<S / S<0\end{array}$ & $\begin{array}{l}22131 \\
22132 \\
22140\end{array}$ & $\begin{array}{l}\mathrm{C} \\
\mathrm{C}\end{array}$ \\
\hline & & Test & $\begin{array}{l}\text { Self-term A } \\
\text { Self-term A } \\
\text { Self-term B } \\
\text { Self-term B }\end{array}$ & $\begin{array}{l}S<0 \\
0<S \\
S<0 \\
0<S\end{array}$ & $\begin{array}{l}22231 \\
22232 \\
22241 \\
22242\end{array}$ & \\
\hline & & Fixed & $\begin{array}{l}\text { Self-term } \\
\text { Self-term } \\
\text { Exhaustive }\end{array}$ & $\begin{array}{l}\mathrm{S}, 0 \\
0, S \\
0<\mathrm{S} / \mathrm{S}<0\end{array}$ & $\begin{array}{l}22324 \\
22325 \\
22310\end{array}$ & $\begin{array}{l}\mathbf{B} \\
\mathbf{A} \\
\mathbf{D}\end{array}$ \\
\hline
\end{tabular}

compared. Based on the assumption that subjects are able to evoke or hold a veridical image of the stimulus from the abstracted physical representation in memory (e.g., Posner, 1967; Shepard \& Chipman, 1970), it was assumed that the subject could "overlay" a representation of the memory figure on the test stimulus (e.g., Kosslyn \& Pomerantz, 1977) so that their centers of mass corresponded (centered); this manipulation, or analog process, would be comparable to use of the afterimage, since the stimuli were presented on the screen with their centers aligned. It was assumed that decision reaction speed would be based on the size of the area of noncorrespondence. Assuming that response speed would be inversely related to the amount of nonoverlapping area, different models were created to reflect response decision processes based on the area of the "largest" nonoverlapping segment, "total" area of noncorrespondence, or on the "mean" area of noncorrespondence (total area divided by the number of nonoverlapping segments) between the memory and test figures. Several of the areas of noncorrespondence between cells in the matrix of each analogtemplate model were only slightly different from each other. In order not to discard a model by interpreting it too rigorously (literal) to be reflected in the observed data, ranks which had been based upon area differences within $1 \mathrm{sq} \mathrm{cm}$ of each other were given the same average rank in order to create a set of less strict models (modified).

Feature-parallel. The parallel feature abstraction models we tested are deterministic versions which assume that the feature analyzers are operationally parallel and work independently of one another (Neisser, 1967). The memory representation of the stimulus is a list of features which are accessed and simultaneously compared to the features of the test figure. It is assumed that all the comparisons between the memory and test form commence simultaneously. Two groups of models were further differentiated, depending on whether it was assumed that the subject responded as soon as a feature of difference was noted (self-terminating) or only after all relevant features had been compared (exhaustive). A further refinement of the models was required depending on whether it was assumed that the size comparison was faster $(S<O)$, slower $(O<S)$, or undifferentiable $(S=O)$ from the orientation comparison in processing speed.

Feature-serial. The deterministic serial models differ from the parallel models in the assumption that multidimensional stimuli are compared attribute by attribute, sequentially, one comparison being complete before the next is begun. Three variations of this model were defined. Under the fixed serial model, it was assumed that features were always tested in the same order, either size $(S, O)$ or orientation $(\mathrm{O}, \mathrm{S})$ was compared first. Different models were also derived if it was assumed that the size comparison was faster $(\mathrm{S}<\mathrm{O})$ or slower $(\mathrm{O}<\mathrm{S})$ than the orientation comparison. Two further models were defined in which some form of preattentive processing of either the memory (memory) or test (test) stimuli was assumed to have occurred. In these models the value of a dimension of either the memory or test stimulus was assumed to in- 
fluence the order in which features were compared. All of these subprocesses were of the self-terminating variety. In one subprocess, the subject compared orientation first if the stimulus was small and size first if it was large (self-term A). The other subprocess (self-term B) assumed just the reverse; the subject compared size first if the stimulus was small, and orientation if it was large.

The determination of the ordinal rank of the cells was done separately for each row of a model matrix to force the row sums to the same value, 21 , the sum of the natural ranks for six decisions. The difference between row pairings was calculated using the assumptions of each model, and then each of the six cells in a row were ranked from fastest (1) to slowest (6). Tie ranks were given the average of their natural rank positions. This process created a 7 by 7 matrix model of predictions with the seven "yes" or identity pair cells unfilled. One matrix of 42 ranked cells was derived for each of the unique models. Four of the 15 unique model matrices created are shown with their theory model numbers in Figure 2.

\section{Raw Data to Ordinal Summary Conversion}

After the models had been expressed in rowranked matrix format, we needed to summarize the
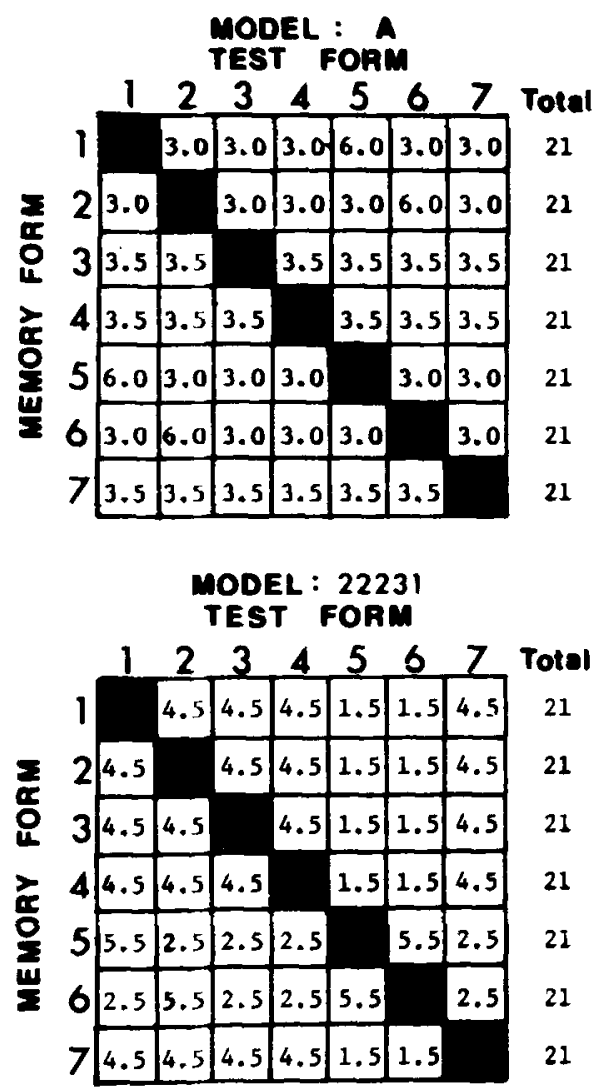

raw data across subjects into the same format. We wished to equate the relative contribution of each subject to the final across-subjects summary data matrix. Simple removal of each subject's mean response time from their data would place all subjects on the same baseline; however, the more variable a subject's times were, the greater would be their contribution to the summary, since the range of the more variable subjects' scores would be considerably greater. To overcome this problem, the response times in each trial block of each subject were normalized using the standard deviation of the reaction times of that subject's trial block. In this manner, the raw millisecond response matrix for each trial block of every subject was converted to a normalized matrix with a mean of 0 and a standard deviation of 1. The normalized subject matrices created were then averaged together. In this way, three separate summary averaged normalized response matrices were created across the subjects, one for each trial block and an overall matrix across both trial blocks. The normalized matrix across trial blocks is presented in Table 3. The normalized summary matrix and the raw millisecond summary matrix across trial blocks correlate .95 with each other.

Every row of each summary matrix of normalized

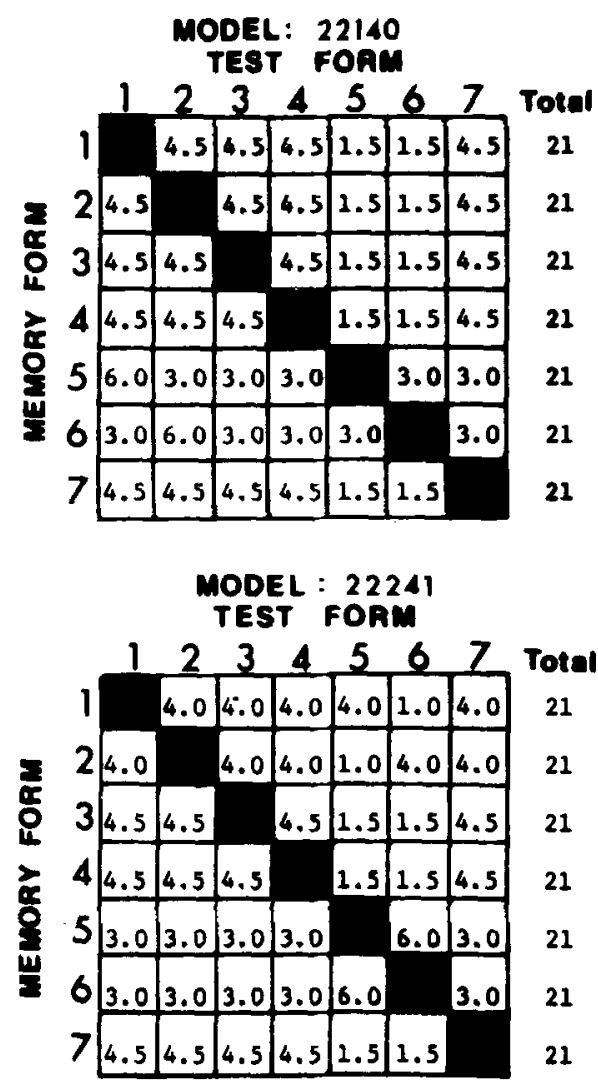


Table 3

Normalized Data Matrix Across Trial Blocks

\begin{tabular}{crccccrr}
$\begin{array}{c}\text { Mem- } \\
\text { ory }\end{array}$ & \multicolumn{7}{c}{ Test Form } \\
\cline { 2 - 8 } Form & 1 & \multicolumn{1}{c}{2} & \multicolumn{1}{c}{3} & \multicolumn{1}{c}{4} & \multicolumn{1}{c}{5} & \multicolumn{1}{c}{6} & \multicolumn{1}{c}{7} \\
\hline 1 & & .093 & .043 & .089 & .597 & -.056 & -.103 \\
2 & .125 & & -.170 & .003 & -.526 & .309 & .070 \\
3 & .184 & .008 & & .455 & -.153 & -.123 & -.135 \\
4 & -.020 & .040 & -.121 & & -.203 & -.283 & .149 \\
5 & .120 & -.053 & .013 & -.089 & & .539 & -.365 \\
6 & -.218 & .512 & .014 & -.070 & .204 & & -.220 \\
7 & .029 & -.013 & .040 & -.088 & -.223 & -.403 & \\
\hline
\end{tabular}

scores was then ranked. Two normalized response scores in a matrix row which differed by more than the selected Z-score value were given different ranks; scores which did not differ by this amount were given the average of their appropriate ranks. Each cell of a row was ranked relative to all other cells; therefore, each cell acted as the anchor or starting point on one "pass." The six sets of row ranks derived from each pass were then accumulated and averaged by cell, creating a single row of six ranked cells. This procedure eliminated the problem of arbitrarily "breaking apart" two scores which were in reality close together in value but just on opposite sides of the Z-score being used. Each time the size of the $Z$-score value was increased, a new matrix whose ordinal ranks were slightly more constrained was created. This sliding scale provided an exact system for inducing ranks within the data by using an increasingly more stringent decision rule. Therefore, for each summary matrix, a series or family of matrices was derived, with : xch matrix of the family differing only by the Z-score value used to rank the differences between the cells of its rows. The observed data matrices were constrained, as were the model matrices, to sum to 21 across a row or memory form.

\section{Model to Subject Summary Correlations}

A correlation was computed for each of the 15 matrix models against each summary matrix series. It was expected that the correlations of descriptive models to the subject matrix summaries would increase as the Z-score level was relaxed, until that point when spurious ranks were induced. The statistic used was the Pearson product moment correlation. Although our data are in the form of ranks across a row, the correlation is computed using all cells in all seven rows of a matrix; this, combined with the allowance of tie ranks, makes the Pearson the more proper statistic (Hays, 1973). The results of the Pearson correlations were compared to those using the Spearman rank correlation coefficient, the Kendall tau coefficient, and several other less known indices. All these techniques derived essentially the same results. Our correlation is, then, a statement of the fraction of the total variance in the paired 42 cell comparisons that is explained by the model.

Figure 3 graphically depicts the correlation curves of the three models which obtained the highest correlation coefficients to the observed subject summary matrix across trial blocks; also included is the curve of the group model $\mathrm{A}$, the group model of highest correlation. All of the curves show low correlations when the decision Z-score level, expressed as a probabilities on the $x$ axis, was set so high that the cells in the observed data were all given the same rank. As the $\alpha$ level was relaxed and smaller Z-score values were used, ranks were introduced and there was an increase in correlation for those models that contained a pattern similar to the pattern of the subject summary matrix.

It is evident from the curves that of all the theories tested, model 22241 shows a consistently higher correlation across the entire family of ranked subject summary data matrices than any of the other models. The highest correlation obtained by this model was at the $.999 \alpha$ level, the least strict level tested, where differential ranks were assigned when there were small differences between cells. This .68 correlation is highly significant, particularly when it is realized that the correlation was computed on stepwise data or ranks, $\mathrm{p}<.001$. A simple correlation between the ranks of this model to the raw reaction time matrix across trial blocks yield a .55 correlation, $\mathrm{p}<.001$. The next two most descriptive models also obtained their highest correlations at the least strict level tested. Model 22231 obtained a .60 and model 22140 a .52 correlation, $\mathrm{p}<.001$. Group model

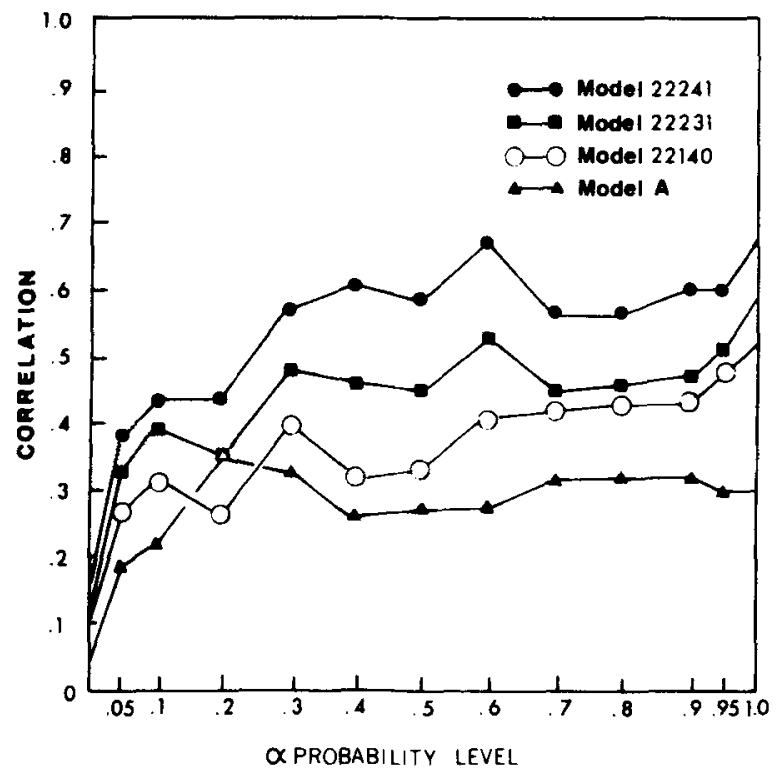

Figure 3. Correlations of the most descriptive models atross trial blocks. 
A yielded a .35 correlation at the $.20 \alpha$ level, $\mathrm{p}<.01$. The maximum correlation achieved by the template models we tested was .43 for model 12120 at the .99 $\alpha$ level, $\mathrm{p}<.001$.

The model of second highest descriptive ability, 22231 , correlates .83 with the most descriptive model, 22241, while the third most descriptive model, 22140 , correlates .55 with model 22241 . The descriptive ability of these models appears to be related to their degree of correlation with model 22241. Of the models tested, the two models of highest correlation are identical in their first three primary assumptions (222); both assume that features are abstracted and then sequentially compared until a feature of difference is determined.

As noted above, there was both a decrease in memory figure study time and a highly significant decrease in response or decision time between replications or trial blocks. Subjects decreased their average response times from 578 to $535 \mathrm{msec}$ from the first to the second block of trials, a 7.4\% decrease. We expected that this decrease indicated a refinement in processing style due to practice, which should be reflected by an increase in the correlation curves of the most descriptive model between replications. To test this, the matrix of model 22241 was correlated separately to the subject summary matrix family for each trial block; these curves are shown in Figure 4. It is apparent that the second block of trials is considerably higher in correlation than the first, obtaining a maximum correlation of .71 at the $.40 \alpha$ level, $\mathrm{p}<.001$. As expected, the refinement in processing style that comes with increased practice was reflected by an even closer match between model 22241 and the subject summary for the second block of trials.

In order to determine if the high correlation of 22241 was an artifact of summing across subjects, separate correlations were also computed individually against each subject's matrix across replications. These correlations were then averaged across subjects and compared. The two most descriptive feature models were again 22241 and 22231. This result supports the inference that the subject summary matrices were reflective of the processing patterns of the individual subjects.

As a final check on individual cognitive style, the response of subjects on a postsession questionnaire on how they "mentally" did the task were scored by two raters who defined each subject as either a template or a feature processor. There were no interrater disagreements on these categorizations, with 13 of the subjects classified as using template and 11 feature processing strategies.

A chi square was computed, comparing each subject's introspective processing style against the model, template or feature, which correlated most highly with their individual summary matrix across

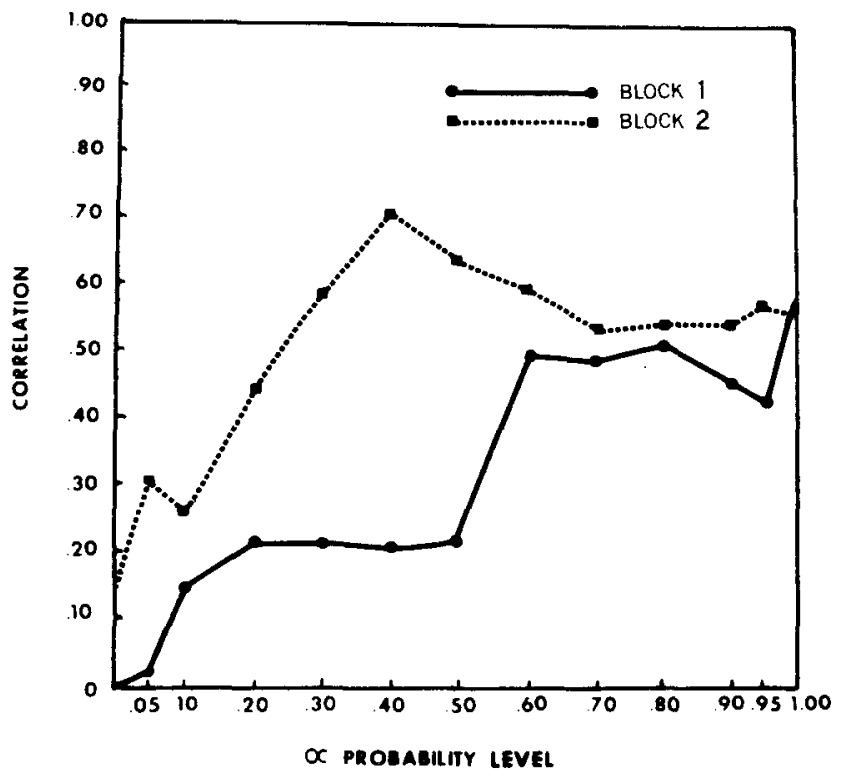

Figure 4. Correlations of model 22241 by trial block.

trial blocks. Of the 24 subjects, only 5 correlated most highly with a template model. The analysis did not obtain significance, $\chi^{2}(1)=.02$, indicating that the data of template processing subjects was not being masked by use of across-subject summary data matrices. As an additional check, the process was repeated for each subject's next most descriptive model, and again the result did not obtain significance, $\chi^{2}(1)=.22$. Apparently, introspective judgments by subjects as to their own processing styles did not match the processing strategy indicated by their pattern of responses.

As a test of model development, a new model was created by a simple averaging of the predictions of our two most descriptive models, 22241 and 22231. This new improved model retains the feature, serial, self-terminating assumptions where the size comparison is faster than the orientation comparison, but now makes the order in which features are processed an equally weighted average as to which attribute will first be selected for processing. In this way, weighted feature testing models can be created which have probabilistic assumptions in either or both feature testing order and decision processing speed. Although it was expected that the maximal correlation we would obtain would lie between the .68 and .60 correlations found for the parent models, this derived model obtained a surprising .87 correlation at the $.90 \alpha$ level. This model obtained higher correlations than any other model, including 22241, at all probability levels. Interestingly, this model is a more definitive version of the model given support in the test figure decision time analysis section of this paper. Although both analyses came to basically the same conclusion, we feel the more extensive 
model testing procedure described above provides for a more complete test of a theory's assumptions. Can we then say that nonidentity processing is based on a serial feature testing process? Unfortunately, no; the ability of certain classes of parallel and serial feature testing models to mimic each other's deductions has been all too adequately pointed out by Townsend (1971b).

A subsequent analysis using our correlation technique found that a simple model in which only the cells in which both sizes and orientation differed between the pairs were ranked faster, obtained a .68 correlation at the $.999 \alpha$ level on the subject matrix summary across trial blocks. This correlation is identical to the maximum correlation obtained by serial model 22241 on the same summary matrix. As concluded in the decision response times section above, we have only been able to show that pairs that differed in two ways were discriminated faster than pairs differing in one. However, we have demonstrated that none of the template, serial or parallel feature exhaustive, or serial or parallel feature selfterminating models with fixed processing order or time assumptions we tested were as adequate as models with less deterministic assumptions.

\section{EXPERIMENT 2}

As discussed above, the six to one ratio of "no" to "yes" responses in the first experiment may have obscured the natural recognition speed relationship between identity and nonidentity responses. This experiment was designed to circumvent that problem by equating the number of "yes" and "no" responses in a testing session.

\section{Method}

For any subject, increasing the number of "yes" pairings in order to equate the number of identity and nonidentity responses increases the length of a session beyond reasonable limits. Since it was believed desirable to take all data at one sitting to minimize rehearsal effects, it was necessary to decrease the total number of trials in a session to a manageable level. Even with the number of trials decreased and the number of identity and nonidentity responses equated, on the identity trials the mernory form would be consistently paired with itself while on the nonidentity trials it could be paired with each of the six other forms. This inequality might bias the subject toward "yes" responding if an expectancy was developed for the more consistent identity pairing (e.g., Williams, 1972). To overcome this difficulty, all subjects received the appropriate seven identity pairs, and for the nonidentity pairings they also received seven consistent pairings.

Since each subject would receive only 7 of the $\mathbf{4 2}$ possible nonidentity pairs, pair assignment to subjects was given careful consideration. The types of differences and relationships between form pairs experienced by the subjects were equated. Each subject was assigned nonidentity pairs which were mirror images, differed in orientation only, size only, and in both size and orientation.

\section{Subjects}

The subjects utilized in this study were also volunteers from undergraduate courses at the University of California, Riverside.
All subject requirements were as in the first experiment. The 12 subjects ranged in age from 19.1 to 46.8 years, with a mean age of 25.0 years.

\section{Apparatus and Procedure}

All equipment utilized for this study was identical to that in the first experiment. The practice trials were changed to individualize the nonidentity pairs to each subject. The numbers of "yes" and "no" trials were equated in the practice session. The postpractice criterion trials requirement was changed to eight consecutive correct responses, four of which were to identity pairs. Only 2 of the 12 subjects made an error during the criterion training trials.

Three random blocks of trials were taken successively, consisting of seven correct "yes" and seven correct "no" responses in each block. As before, any incorrect responses were retaken within the body of the regular session. The voice key triggering speeds of the "yes" and "no" responses were computed individually, as outlined above, for every subject and removed from their decision reaction time scores. On each trial, both scanning time of the memory form and recognition reaction time to the test form were recorded. An average of only 1.1 errors was made in a session over the $\mathbf{4 2}$ responses of each subject.

\section{Results and Discussion}

The comparison between identity and nonidentity response speed found the "yes" to be significantly faster than the "no" responses, $F(1,10)=19.86$, $\mathrm{p}<.01$. The average "yes" response with verbal reaction time removed was $190 \mathrm{msec}$, while the average "no" response was $251 \mathrm{msec}$, a rather large 61 -msec difference. The average raw response speed was $614 \mathrm{msec}$ for the "yes" and 694 for the "no" response.

A simple one-way analysis of variance of only the "yes" responses found that the identity response speeds did not differ significantly by form, $F(6,60)$ $=2.08, \mathrm{p}>.05$. Although this analysis does approach significance, the lack of a significant finding is not contradictory of a simple template or wholistic processing model for identity decisions, which is supported by the significantly faster identity processing found in this and other studies (e.g., Nickerson, 1967; Smith \& Nielsen, 1970).

It should be noted that our results, using pairs equated for familiarity and response type in a design similar to Williams (1972), did demonstrate faster identity processing. This result contradicts Williams, making his claim that Nickerson's (1972) finding of shorter reaction times for some responses were caused by differential practice effects less tenable.

\section{CONCLUSION}

The original purpose of this study was to optimize conditions for wholistic template image processing in a sequential recognition decision task and to test a broader range of potential template models than have previously been examined. We believe these goals were realized, although the final result was not in the expected direction. We were unable to demonstrate nonidentity template processing, even under 
what we hoped would be ideal conditions for such a process to take place.

The significantly faster reaction times for identity responses found in Experiment 2 coupled with the nonidentity model testing of Experiment 1, provide support for a dual process model similar to that proposed by Bamber (1969). In this model, identity and nonidentity testing proceed in parallel, with identity testing possibly based on a fast wholistic comparison process and nonidentity testing based on a slower form of either serial or parallel, self-terminating, feature comparison.

Although the nonidentity decision process was apparently not accomplished by a template comparison, our results do not necessarily conflict with prior findings of wholistic image utilization. It is still possible that the figure in memory was being held as a whole but that the recognition decision process required features to be abstracted from the memory image and compared serially to the test stimulus until a mismatch was determined.

\section{REFERENCES}

BAMBER. D. Reaction times and error rates for "same"."different" judgments of multidimensional stimuli. Perception \& Psychophysics, 1969, 6, 169-174.

BEHAR, I. On the relation between response uncertainty and reaction time in category judgements. Perceptual and Motor Skills, 1963, 16, 595-596.

COOPER, L. A. Mental rotation of random two-dimensional shapes. Cognitive Psychology, 1975, 7. 20-43.

Cooper, L. A.. \& ShePaRd, R. N. The time required to prepare for a rotated stimulus. Memory \& Cognition, 1973, 1, 246.250.

Downing. B. D., \& Gossman, J. R. Parallel processing of multidimensional stimuli. Perception \& Psychophysics, 1970 , 8. 57.60

Egeth, H. E. Parallel versus serial processes in multidimensional stimulus discrimination. Perception \& Psychophysics, 1966. 1. $245-252$.

Garner. W. R., \& Felfoldy, G. L. Integrality of stimulus dimensions in various types of information processing. Cognitive Psychology, 1970, 1, 225-241.

Geyer, L. H., \& DeW ALd, C. G. Feature lists and confusion matrices. Perception \& Psychophysics, 1973, 14, 471-482.
Hawkins, H. L. Parallel processing in complex visual discrimination. Perception \& Psychophysics, 1969, 5, 56-64.

Hays, W. L. Statistics for the social sciences (2nd ed.). New York: Holt, Rinehart, and Winston, 1973.

Hock, H. S. The effects of stimulus structure and familiarity on same-different comparison. Perception \& Psychophysics, 1973. 14. 413-420.

KosSLYN, S. M. Information representation in visual images. Cognitive Psychology, 1975, 7, 341-370.

Kosslyn, S. M., \& Pomerantz, J. R. Imagery, propositions, and the form of internal representations. Cognitive Psychology, $1977,9.52-76$

Nersser, U. Cognitive psychology. New York: Appleton-CenturyCrofts, 1967.

Nickerson, R. S. "Same".-"different" response times with multi-attribute stimulus differences. Perceptual and Motor Skills, 1967, 24, 543-554.

Nickerson. R. S. Auditory codability and the short-term retention of visual information. Joumal of Experimental Psychology, 1972, 95, 429-436.

POSNER. M. I. Short-term memory systems in human information processing. Acta Psychologica, 1967, 27, 267-284.

Posner, M. I., Boies, S. J., Eichelman. W. H., \& Tayor. R. L. Retention of visual and name codes of single letters. Journal of Experimental Psychology Monograph, 1969, 79, (1, Pt. 2).

Shepard, R. N.. \& Chipman, S. Second-order isomorphisms of internal representations: Shapes of states. Cognitive Psychology. 1970, 1, 1-17.

Shepard, R. N., \& Metzler, J. Mental rotation of threedimensional objects. Science, 1971, 171, 701-703.

Silverman. W. P. The perception of identity in simultaneously presented complex visual displays. Memon \& Cognition. 1973. 1. 459-466.

Silverman, W. P., \& Goldberg. S. L. Further confirmation of same vs. different processing differences. Perception \& Psychophysics, 1975, 17, 189-193.

Smith, E. E., \& Nielsen, G. Representations and retrieval processes in short-term memory: Recognition and recall of faces. Journal of Experimental Psychology, 1970, 85, 397-405.

Townsend. J. T. Theoretical analysis of an alphabetic confusion matrix, Perception \& Psychophysics, 1971. 9. 40-49. (a)

Townsend, J. T. A note on the identifiability of parallel and serial processes. Perception \& Psychophysics. 1971, 10. 161-163. (b)

Williams. J. D. Effects of practice with controlled stimulus pairs on same-different judgements. Joumal of Experimental Psychology, 1972, 96, 73-77.

(Received for publication April 22, 1977 ; accepted June 10. 1977.) 